\title{
Subthreshold stochastic resonance: Rectangular signals can cause anomalous large gains
}

\author{
Jesús Casado-Pascual,* José Gómez-Ordóñez, and Manuel Morillo \\ Física Teórica, Universidad de Sevilla, Apartado de Correos 1065, Sevilla 41080, Spain \\ Peter Hänggi \\ Institut für Physik, Universität Augsburg, Universitätsstraße 1, D-86135 Augsburg, Germany
}

(Received 27 May 2003; published 17 December 2003)

\begin{abstract}
The main objective of this work is to explore aspects of stochastic resonance (SR) in noisy bistable, symmetric systems driven by subthreshold periodic rectangular external signals possessing a large duty cycle of unity. Using a precise numerical solution of the Langevin equation, we carry out a detailed analysis of the behavior of the first two cumulant averages, the correlation function, and its coherent and incoherent parts. We also depict the nonmonotonic behavior versus the noise strength of several SR quantifiers such as the average output amplitude, i.e., the spectral amplification, the signal-to-noise ratio, and the SR gain. In particular, we find that with subthreshold amplitudes and for an appropriate duration of the pulses of the driving force, the phenomenon of stochastic resonance is accompanied by SR gains exceeding unity. This analysis thus sheds light on the interplay between nonlinearity and the nonlinear response, which in turn yields nontrivial unexpected SR gains above unity.
\end{abstract}

DOI: 10.1103/PhysRevE.68.061104

PACS number(s): 05.40.-a, 05.10.Gg, 02.50.-r

\section{INTRODUCTION}

Most studies on the phenomenon of stochastic resonance (SR) in dynamical systems have been devoted to systems driven by sinusoidal terms (see [1-5] for reviews). Several analytical approximations have been put forward to explain SR. In the approach of McNamara and Wiesenfeld [6], the Langevin dynamics is replaced by a reduced two-state model that neglects the intrawell dynamics. The general ideas of linear response theory (LRT) have been applied to situations where the input amplitude is small [2,7-13]. In [11,13] the Floquet theory has been applied to the corresponding Fokker-Planck description. For very low input frequencies, an adiabatic ansatz has been invoked [13]. Even though these alternative analytical approaches provide an explanation of SR for different regions of parameter values, their precise limits of validity remain to be determined. In recent work $[14,15]$, we explored the validity of LRT for sinusoidal and multifrequency input signals with low frequency. Our results indicate a breakdown of the LRT description of the average behavior for low frequency, subthreshold amplitude inputs.

Several quantifiers have been used to characterize SR in noisy, continuous systems. The average output amplitude, or the spectral amplification (SPA), has been studied in Refs. $[11,13]$ and the phase of the output average in Refs. [16-18], respectively. Those parameters as well as the signal-to-noise ratio (SNR) [6], exhibit a nonmonotonic behavior with the noise strength which is representative of SR. An important quantity is the SR gain, defined as the ratio of the SNR of the output over the input SNR. It has been repeatedly pointed out that the SR gain cannot exceed unity as long as the system operates in a regime described by LRT $[19,20]$. Beyond LRT there exists no physical reason that prevents the SR gain to

*Electronic address: jcasado@us.es; http://numerix.us.es be larger than 1, as has been demonstrated in [21] for superthreshold sinusoidal input signals, and in analog experiments in $[22,23]$ for subthreshold input signals with many Fourier components and a small duty cycle [20].

In this work, we will make use of numerical solutions of the Langevin equation following the methodology in [20] to analyze SR in noisy bistable systems, driven by a periodic piecewise constant signal with two amplitude values of opposite signs (rectangular signal) (see Fig. 1). There are several relevant time scales in the dynamics of these systems: (i) $t_{\text {asym }}$, the time interval within each half period of the driving force, during which the diffusing particle sees an asymmetric constant two-well potential; (ii) $t_{\text {inter }}$, the time scale associated with the interwell transitions in both directions; and (iii) $t_{\text {intra }}$, the time scale associated with intrawell dynamics. The interwell and intrawell time scales depend basically on the noise strength $D$ and the amplitude of the driving term. The dependence of these two time scales on those parameters is certainly very different, being more pronounced for $t_{\text {inter }}$. Typically, for the range of parameter values associated with SR, the intrawell time scale is shorter than the interwell one. For small values of $D$, Kramers' formula provides an esti-

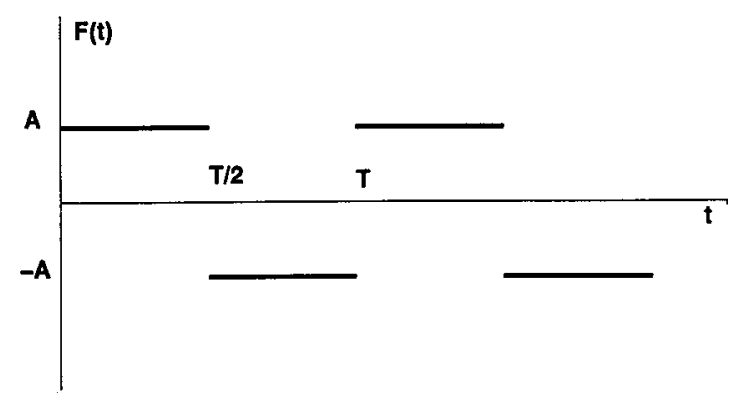

FIG. 1. Sketch of a rectangular periodic signal with duty cycle 1, amplitude $A$, and period $T$. 
mate for $t_{\text {inter }}^{-1}$ [24], while $t_{\text {intra }}^{-1}$ is basically of the order of the curvatures at the bottom of the wells.

We will evaluate the long-time average behavior of the output and the second cumulant. These two quantities were studied some time ago by two of us [25] for periodic rectangular driving signals. Here, we will further extend our work to the analysis of the correlation function and its coherent and incoherent parts. The knowledge of all these quantities provides very useful information for the explanation of SR, as indicated by the nonmonotonic behavior with the noise strength of the output amplitude and the SNR. In particular, the knowledge of the incoherent part of the correlation function is of outmost importance for a correct determination of the SNR. Furthermore, for a given subthreshold amplitude, we will demonstrate that, if there exists a range of noise values such that $t_{\text {inter }}$ is shorter than $t_{\text {asym }}$, then it is possible to observe stochastic amplification and, simultaneously, SR gains larger than unity. This is strictly forbidden by linear response theory, as we have recently shown [20]. Thus, the simultaneous appearance of stochastic amplification and SR gains above 1 implies a strong violation of linear response theory.

\section{MODEL SYSTEM AND SR QUANTIFIERS}

Let us consider a system characterized by a single degree of freedom $x$ subject to the action of a zero average Gaussian white noise with $\langle\xi(t) \xi(s)\rangle=2 D \delta(t-s)$ and driven by an external periodic signal $F(t)$ with period $T$. In the Langevin description, its dynamics is generated by the equation

$$
\dot{x}(t)=-U^{\prime}[x(t)]+F(t)+\xi(t) .
$$

The corresponding linear Fokker-Planck equation (FPE) for the probability density $P(x, t)$ reads

$$
\frac{\partial}{\partial t} P(x, t)=\hat{\mathcal{L}}(t) P(x, t)
$$

where

$$
\hat{\mathcal{L}}(t)=\frac{\partial}{\partial x}\left[U^{\prime}(x)-F(t)+D \frac{\partial}{\partial x}\right] .
$$

In the expressions above, $U^{\prime}(x)$ represents the derivative of the potential $U(x)$. In this work, we will consider a bistable potential $U(x)=-x^{2} / 2+x^{4} / 4$. The periodicity of the external driving $F(t)$ allows its Fourier series expansion in the harmonics of the fundamental frequency $\Omega=2 \pi / T$, i.e.,

$$
F(t)=\sum_{n=1}^{\infty}\left[f_{n} \cos (n \Omega t)+g_{n} \sin (n \Omega t)\right],
$$

with the Fourier coefficients $f_{n}$ and $g_{n}$ given by

$$
\begin{aligned}
& f_{n}=\frac{2}{T} \int_{0}^{T} d t F(t) \cos (n \Omega t), \\
& g_{n}=\frac{2}{T} \int_{0}^{T} d t F(t) \sin (n \Omega t) .
\end{aligned}
$$

Here, we are assuming that the cycle average of the external driving over its period equals zero. In this work, we will focus our attention on multifrequency input forces with "rectangular" shape given by

$$
F(t)= \begin{cases}A, & 0 \leqslant t<T / 2 \\ -A, & T / 2 \leqslant t<T,\end{cases}
$$

as sketched in Fig. 1. The external force remains constant at a value $A$ during each half period and changes sign for the second half of the period. The duty cycle of a signal can be defined in a variety of ways. In this work, we will follow the definition used by Gingl et al. [22,23]. Thus, by "duty cycle" we mean the ratio of the time span during which the signal is nonzero to the total period of the signal. Consequently, the rectangular signal in Eq. (6) possesses a duty cycle of unity. Notice that the duty cycle does not uniquely characterize a periodic signal.

The two-time correlation function $\langle x(t+\tau) x(t)\rangle_{\infty}$ in the limit $t \rightarrow \infty$ is given by

$$
\begin{aligned}
&\langle x(t+\tau) x(t)\rangle_{\infty} \\
& \quad=\int_{-\infty}^{\infty} d x^{\prime} x^{\prime} P_{\infty}\left(x^{\prime}, t\right) \int_{-\infty}^{\infty} d x x P_{1 \mid 1}\left(x, t+\tau \mid x^{\prime}, t\right),
\end{aligned}
$$

where $P_{\infty}(x, t)$ is the time-periodic, asymptotic long-time solution of the FPE and the quantity $P_{1 \mid 1}\left(x, t+\tau \mid x^{\prime}, t\right)$ denotes the two-time conditional probability density that the stochastic variable will have a value near $x$ at time $t+\tau$ if its value at time $t$ was exactly $x^{\prime}$. It can been shown [2,13] that, in the limit $t \rightarrow \infty$, the two-time correlation function $\langle x(t$ $+\tau) x(t)\rangle_{\infty}$ becomes a periodic function of $t$ with the period of the external driving. Then, we define the one-time correlation function $C(\tau)$ as the average of the two-time correlation function over a period of the external driving, i.e.,

$$
C(\tau)=\frac{1}{T} \int_{0}^{T} d t\langle x(t+\tau) x(t)\rangle_{\infty} .
$$

The correlation function $C(\tau)$ can be written exactly as the sum of two contributions: a coherent part $C_{c o h}(\tau)$, which is periodic in $\tau$ with period $T$, and an incoherent part which decays to 0 for large values of $\tau$. The coherent part $C_{c o h}(\tau)$ is given by $[2,13]$

$$
C_{c o h}(\tau)=\frac{1}{T} \int_{0}^{T} d t\langle x(t+\tau)\rangle_{\infty}\langle x(t)\rangle_{\infty},
$$

where $\langle x(t)\rangle_{\infty}$ is the average value evaluated with the asymptotic form of the probability density $P_{\infty}(x, t)$.

According to McNamara and Wiesenfeld [6], the output SNR is defined in terms of the Fourier transform of the coherent and incoherent parts of $C(\tau)$. As the correlation func- 
tion is even in time and we evaluate its time dependence for $\tau \geqslant 0$, it is convenient to use its Fourier cosine transform, defined as

$$
\begin{aligned}
\widetilde{C}(\omega) & =\frac{2}{\pi} \int_{0}^{\infty} d \tau C(\tau) \cos (\omega \tau), \\
C(\tau) & =\int_{0}^{\infty} d \omega \widetilde{C}(\omega) \cos (\omega \tau) .
\end{aligned}
$$

The value of the output SNR is then obtained from

$$
R_{\text {out }}=\frac{\lim _{\epsilon \rightarrow 0^{+}} \int_{\Omega-\epsilon}^{\Omega+\epsilon} d \omega \widetilde{C}(\omega)}{\widetilde{C}_{\text {incoh }}(\Omega)} .
$$

Note that this definition of the SNR differs by a factor of 2, stemming from the same contribution at $\omega=-\Omega$, from the definitions used in earlier works $[2,13]$. The periodicity of the coherent part gives rise to $\delta$ function peaks in the spectrum. Thus, the only contribution to the numerator in Eq. (11) stems from the coherent part of the correlation function. The evaluation of the SNR requires the knowledge of the Fourier components of $C_{c o h}(\tau)$ and $C_{\text {incoh }}(\tau)$ at the fundamental frequency of the driving force. Thus, rather than knowledge of the entire Fourier spectrum, only two well defined numerical quadratures are needed. These are

$$
R_{o u t}=\frac{Q_{u}}{Q_{l}}
$$

where

$$
Q_{u}=\frac{2}{T} \int_{0}^{T} d \tau C_{c o h}(\tau) \cos (\Omega \tau)
$$

and

$$
Q_{l}=\frac{2}{\pi} \int_{0}^{\infty} d \tau C_{\text {incoh }}(\tau) \cos (\Omega \tau)
$$

The SNR for an input signal $F(t)+\xi(t)$ is given by

$$
R_{i n p}=\frac{\pi\left(f_{1}^{2}+g_{1}^{2}\right)}{4 D}
$$

The SR gain $G$ is consequently defined as the ratio of the SNR of the output to the SNR of the input, namely,

$$
G=\frac{R_{o u t}}{R_{\text {inp }}}
$$

\section{NUMERICAL SOLUTION}

Stochastic trajectories $x^{(j)}(t)$ are generated by numerically integrating the Langevin equation [Eq. (1)] for every realization $j$ of the white noise $\xi(t)$, starting from a given initial condition $x_{0}$. The numerical solution is based on the algorithm developed by Greenside and Helfand [26,27] (consult also the Appendix in Ref. [20]). After allowing for a relaxation transient stage, we start recording the time evolution of each random trajectory for many different trajectories. Then we construct the long-time average value,

$$
\langle x(t)\rangle_{\infty}=\frac{1}{N} \sum_{j=1}^{N} x^{(j)}(t)
$$

and the second cumulant

$$
\left\langle x^{2}(t)\right\rangle_{\infty}-\langle x(t)\rangle_{\infty}^{2}=\frac{1}{N} \sum_{j=1}^{N}\left[x^{(j)}(t)\right]^{2}-\left[\frac{1}{N} \sum_{j=1}^{N} x^{(j)}(t)\right]^{2}
$$

where $N$ is the number of stochastic trajectories considered. We also evaluate the two-time ( $t$ and $\tau$ ) correlation function, i.e.,

$$
\langle x(t+\tau) x(t)\rangle_{\infty}=\frac{1}{N} \sum_{j=1}^{N} x^{(j)}(t+\tau) x^{(j)}(t)
$$

as well as the product of the averages

$$
\langle x(t+\tau)\rangle_{\infty}\langle x(t)\rangle_{\infty}=\left[\frac{1}{N} \sum_{j=1}^{N} x^{(j)}(t+\tau)\right]\left[\frac{1}{N} \sum_{j=1}^{N} x^{(j)}(t)\right] .
$$

The correlation function $C(\tau)$ and its coherent part $C_{c o h}(\tau)$ are then obtained using their definitions in Eqs. (8) and (9), performing the cycle average over one period of $t$. The difference between the values of $C(\tau)$ and $C_{c o h}(\tau)$ allows us to obtain the values for $C_{\text {incoh }}(\tau)$. It is then straightforward to evaluate the Fourier component of $C_{c o h}(\tau)$ and the Fourier transform of $C_{\text {incoh }}(\tau)$ at the driving frequency by numerical quadrature. With that information, the numerator and the denominator for the output SNR [cf. Eqs. (12), (13), and (14)], as well as the SR gain [cf. Eq. (16)], are obtained.

\section{RESULTS}

\section{A. Response to a rectangular driving force with fundamental frequency $\Omega=0.01$}

Consider an external driving of the type sketched in Fig. 1 with parameter values $\Omega=0.01, A=0.25$. This amplitude is well below its threshold value defined, for each driving frequency, as the minimum amplitude that can induce repeated transitions between the minima of $U(x)$ in the absence of noise. For the input considered here, the threshold amplitude is $A_{T} \approx 0.37$. Note that this threshold value for the amplitude increases with increasing driving frequency.

In Fig. 2 we depict with several panels the behavior of the first two cumulants $\langle x(t)\rangle_{\infty}$ and $\left\langle x^{2}(t)\right\rangle_{\infty}-\langle x(t)\rangle_{\infty}^{2}$ for several representative values of $D$ [from top to bottom $D$ $=0.02$ (a), $D=0.04$ (b), $D=0.06$ (c), $D=0.1$ (d), and $D$ $=0.2$ (e)]. Notice that, due to the transients, the time at which we start recording data, $t=0$ in the graphs, does not necessarily coincide with the start of an external cycle. The average is periodic with the period of the driving force, while 


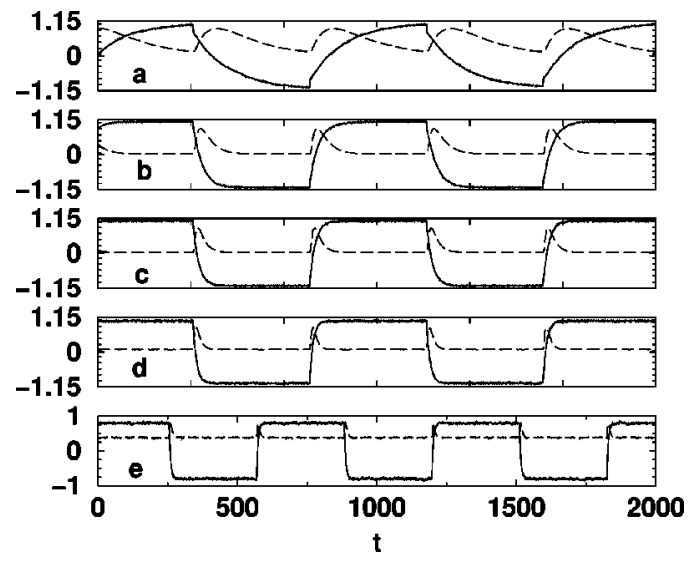

FIG. 2. Time behavior of the average $\langle x(t)\rangle_{\infty}$ (solid lines) and the second cumulant $\left\langle x^{2}(t)\right\rangle_{\infty}-\langle x(t)\rangle_{\infty}^{2}$ (dashed lines) for a rectangular driving force with duty cycle 1 , fundamental frequency $\Omega$ $=0.01$, and subthreshold amplitude $A=0.25$ for several values of the noise strength: $D=0.02$ (a), $D=0.04$ (b), $D=0.06$ (c), $D=0.1$ (d), $D=0.2$ (e). Notice that, due to the transients, $t=0$ in the graphs does not necessarily coincide with the start of an external cycle.

the second cumulant, due to the reflection symmetry of the potential [14], is periodic with a period one-half of the period of the forcing term.

Next we consider the case of small noise intensity $D$ [say, $D=0.02$ as in Fig. 2(a)]. The noise induces jumps between the wells. In each random trajectory, a jump between the wells has a very short duration, but the instants of time at which they take place for the different stochastic trajectories are randomly distributed during a half cycle. At this small noise strength, the jumps are basically toward the lowest minimum. Thus, because of this statistical effect, the average behavior depicts the smooth evolution depicted in Fig. 2(a), without sudden transitions between the wells. The evolution of the second cumulant adds relevant information. The fact that it is rather large during most of a period indicates that the probability density $P_{\infty}(x, t)$ is basically bimodal during most of the external cycle. It is only during very short time intervals around each half period that the probability distribution becomes monomodal around one of the minima, and, consequently, the second cumulant is small. The bimodal character arises from the fact that the noise is so small in comparison with the barrier heights that jumps over the barrier are rather infrequent during each half period.

As the noise strength increases, the time evolution of $\langle x(t)\rangle_{\infty}$ follows closely the shape of the external force [see Figs. 2(b) $-2(\mathrm{~d})]$ for $0.04 \leqslant D \leqslant 0.1]$. This behavior indicates that, for these parameter values, the jumps in the different random trajectories are concentrated within short time intervals around the instants of time at which the driving force switches sign. The second cumulant remains very small during most of a period, except for short time intervals around the switching times of the external driver. Thus, for these intermediate values of $D$, the probability distribution, $P_{\infty}(x, t)$ is basically monomodal, except for small time intervals around the switching instants of time of the periodic driver. Finally, as the noise strength is further increased ( $D$ $>0.1)$, the probability distribution remains very broad most

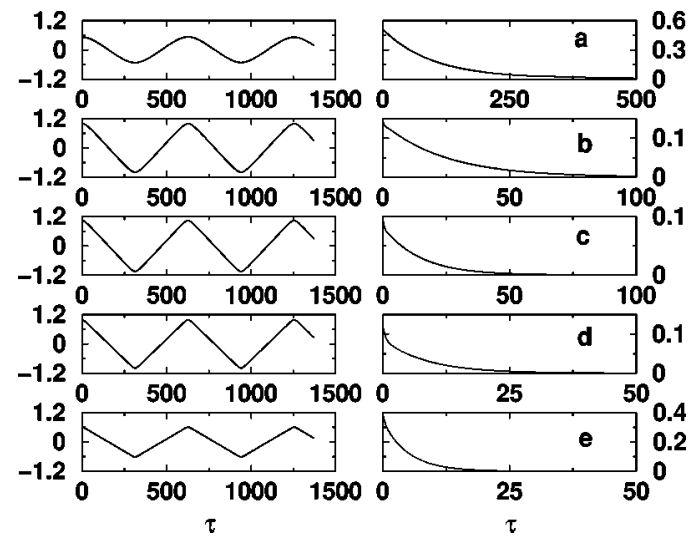

FIG. 3. Time behavior of $C_{c o h}(\tau)$ (left panels) and $C_{\text {incoh }}(\tau)$ (right panels) for a rectangular driving force with duty cycle 1 , fundamental frequency $\Omega=0.01$, and subthreshold amplitude $A$ $=0.25$ for several values of the noise strength: $D=0.02$ (a), $D$ $=0.04$ (b), $D=0.06$ (c), $D=0.1$ (d), $D=0.2$ (e).

of the time. Even though a large majority of random trajectories will still jump over the barrier in synchrony with the switching times of the driver, the noise is so large in comparison with the barrier heights that the probability of crossings over the barrier in both directions cannot be neglected at any time during each half cycle. The probability distribution remains bimodal during a whole period, but asymmetric: the larger fraction of the probability accumulates around the corresponding lower minima of the potential during each cycle. Therefore, the average output amplitude decreases, while the second cumulant depicts plateaus at higher values than for smaller noise strengths [compare in Figs. 2(c) and 2(e)].

In Fig. 3, we plot the coherent (left panels) $C_{c o h}(\tau)$ and incoherent (right panels) $C_{\text {incoh }}(\tau)$ components of the correlation function $C(\tau)$ for the same parameter values as in Fig. 2 . The coherent part shows oscillations with a period equal to that of the driving force. Its shape changes with $D$. The amplitude of the coherent part does not grow monotonically with $D$. Rather, it maximizes at $D \approx 0.06$, which is consistent with the observed behavior of $\langle x(t)\rangle_{\infty}$ in Fig. 2. This is expected as the evaluation of $C_{c o h}(\tau)$ involves only the time behavior of $\langle x(t)\rangle_{\infty}$ at two different instants of time.

Two features of the behavior of $C_{\text {incoh }}(\tau)$ are relevant: its initial value and its decay time. The initial value of the incoherent contribution, $C_{\text {incoh }}(0)$, is given by the cycle average of the second cumulant. It has a nonmonotonic behavior with $D$. For $D=0.02, C_{\text {incoh }}(0)$ is large, consistent with the fact that the second cumulant at this noise strength is appreciably different from 0 during a substantial part of a period. As the value of $D$ increases $(D \leqslant 0.1)$, the value of $C_{\text {incoh }}(0)$ decreases. This is expected as the second cumulant is large just during those small time intervals where most of the forward transitions take place every half period. For still larger values of $D$ there are frequent forward and backward jumps that keep the stationary probability bimodal, and therefore the initial value $C_{\text {incoh }}(0)$ increases. For $D=0.02$, the decay of $C_{\text {incoh }}(\tau)$ is very slow, although the decay time is still shorter than the duration of half a period of the driving force. As $D$ increases, the decay time of the incoherent part be- 


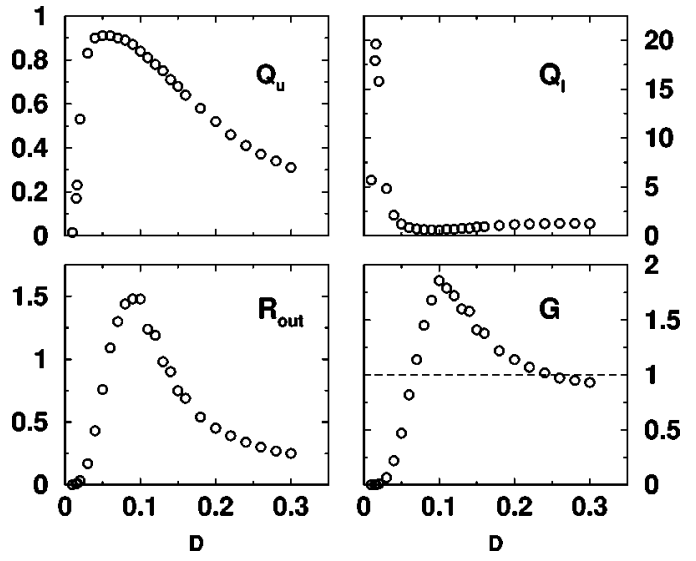

FIG. 4. Dependence on $D$ of several SR quantifiers: the numerator of the SNR $\left(Q_{u}\right)$, its denominator $\left(Q_{l}\right)$, the output SNR $\left(R_{\text {out }}\right)$, and the SR gain $(G)$ for a rectangular driving force with duty cycle 1, fundamental frequency $\Omega=0.01$, and subthreshold amplitude $A$ $=0.25$.

comes shorter. It is worth pointing out that the intrawell noisy dynamics manifests itself in the behavior of $C_{\text {incoh }}(\tau)$. This is most clearly confirmed by noticing the fast initial decay observed in Fig. 3(d). For smaller values of $D$, this feature is masked by the long total relaxation time scale, while for $D=0.2$, the noise strength is so large that there is not a clear-cut separation between inter- and intrawell time scales.

The above considerations allow us to rationalize the behavior of the several quantifiers used to characterize SR. Their behaviors with $D$ for $A=0.25, \Omega=0.01$ are depicted in Fig. 4. It should be noted that the lowest value of the noise strength used in the numerical solution of the Langevin equation is $D=0.01$. For this noise strength, the values of $Q_{u}$ and $R_{\text {out }}$ are very small, although not zero. For even lower noise strengths the task becomes computationally very demanding and expensive, due to the extremely slow decay of the correlations. For $D$ sufficiently small, however, one does expect $Q_{u}$ to be larger than $Q_{l}$, and, consequently, an increase of the numerical $R_{\text {out }}$ as $D$ is lowered.

The quantity $Q_{u}$ defined in Eq. (13) depicts a nonmonotonic dependence on $D$ typical of the SR phenomenon. Its behavior is expected from the dependence of the amplitude of $C_{c o h}(\tau)$ with $D$ in Fig. 3 .

A nonmonotonic behavior with $D$ for the numerically evaluated $Q_{l}$ is also observed. The initial value $C_{\text {incoh }}(0)$ and the decay time of $C_{\text {incoh }}(\tau)$ are important in the evaluation of $Q_{l}$ [see Eq. (14)]. For $D=0.01$, the decay time of the incoherent part of the correlation function is longer than half a period of the driving force, while for $D=0.02$, it is somewhat shorter than $T / 2$. Consistently with Eq. (14), the value of the integral for $D=0.01$ is smaller than for $D$ $=0.02$. As $D$ is further increased, the influence of the cosine factor in Eq. (14) becomes less important as the decay time is much shorter than $T / 2$. The drastic fall in the $Q_{l}$ values observed for $0.02<D<0.1$ is due to the decrease of $C_{\text {incoh }}(0)$ with $D$ [see Fig. 3(a)-3(c)] and the shortening of the decay time. As $D$ is increased further, $C_{\text {incoh }}(0)$ increases and, consequently, $Q_{l}$ also increases slightly.

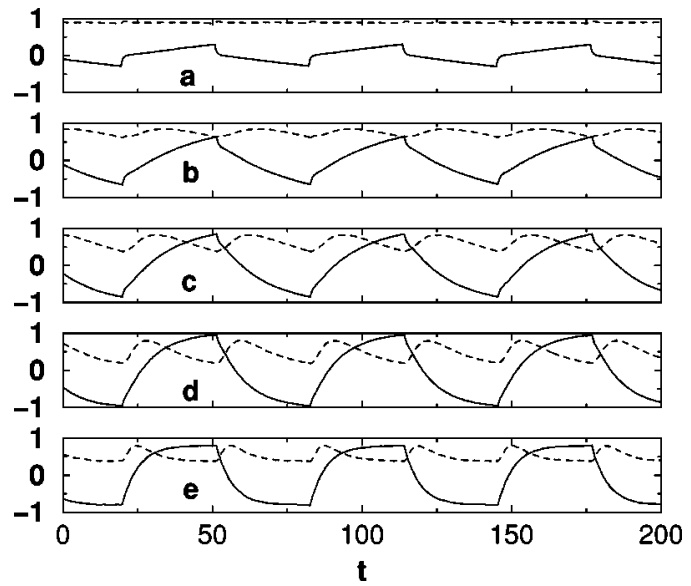

FIG. 5. Same as Fig. 2 but for $\Omega=0.1$.

Taking into account the definition of the SNR [cf. Eq. (12)], its behavior with $D$ is not surprising. The numerically obtained SNR peaks at $D=0.08$, a slightly different value of $D$ from the one at which $Q_{u}$ peaks.

\section{B. Anomalous SR gain behavior for subthreshold driving}

The SR gain is defined in Eq. (16). The numerically determined SR gain shows a most interesting feature: we observe a nonmonotonic behavior versus $D$, with values for the gain exceeding unity for a whole range of noise strengths. This is strictly forbidden within LRT [20]; therefore, the fact that the SR gain can assume values larger than unity reflects a manifestation of the inadequacy of LRT to describe the system dynamics for the parameter values considered.

To rationalize this anomalous SR gain behavior, we notice that the role of the noise in the dynamics is twofold. On the one hand, it controls the decay time of $C_{\text {incoh }}(\tau)$. On the other hand, the noise value is relevant to elucidate whether the one-time probability distribution is basically monomodal or bimodal during most of the cycle and, consequently, it controls the initial value $C_{\text {incoh }}(0)$. As discussed above, if $D$ is small, the decay time is very large compared to $t_{\text {asym }}$, and the one-time probability distribution is essentially bimodal. For large values of $D$, the decay of $C_{\text {incoh }}(\tau)$ is fast enough, and the distribution is also bimodal. The large SR gain obtained here requires the existence of a range of intermediate noise values such that (i) $C_{\text {incoh }}(\tau)$ decays on a much shorter time scale than $t_{\text {asym }}$ and (ii) the one-time probability distribution remains monomodal during most of the external cycle. For example, for $D=0.1$ where the maximum of the SR gain is obtained (see Fig. 4), the decay time of $C_{\text {incoh }}(\tau)$ estimated from an exponential fitting to the behavior depicted in Fig. 3(d) yields a value of approximately 8, while $t_{\text {asym }}=T / 2 \approx 314$.

\section{Response to a rectangular input driver with fundamental frequency $\Omega=0.1$}

As mentioned before, there are several time scales that are important for the phenomenon of stochastic amplification and gain. In the previous subsection we considered an input frequency small enough so that the inequality $t_{\text {asym }}>t_{\text {inter }}$ 


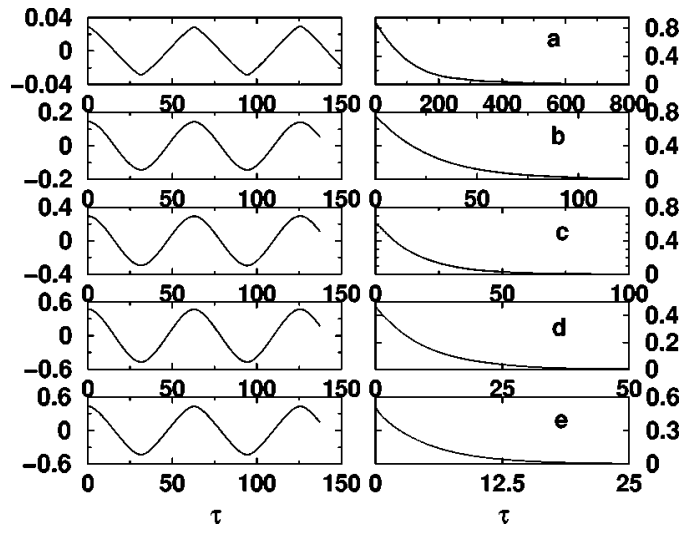

FIG. 6. Same as Fig. 3 but for $\Omega=0.1$.

holds for a range of noise values. Next, we shall analyze the system response to a driving force with fundamental frequency $\Omega=0.1$, ten times larger than in the previous case. We will take the same input amplitude as in the previous case, $A=0.25$, which is still subthreshold. For this input frequency, the threshold value for the amplitude strength is determined by numerically solving the deterministic equation to yield $A_{T} \approx 0.39$.

The behavior of the first two cumulants for several values of the noise strength is depicted in Fig. 5 [from top to bottom $D=0.02$ (a), $D=0.04$ (b), $D=0.06$ (c), $D=0.1$ (d) and $D$ $=0.2(\mathrm{e})]$. For all values of $D$, the second cumulant remains large for most of each half period. By contrast with the lower frequency case, we detect no values of $D$ for which the probability distribution is monomodal for a significant fraction of each half period.

In Fig. 6 the behavior of the coherent (left panels) and incoherent (right panels) parts of the correlation function is presented for (from top to bottom) $D=0.02$ (a), $D=0.04$ (b), $D=0.06$ (c), $D=0.1$ (d), and $D=0.2$ (e). The amplitude of the coherent oscillations shows a nonmonotonic behavior with $D$. The incoherent part has initial values that remain very large in comparison with the corresponding ones for $\Omega=0.01$ (compare with Fig. 3 ), consistently with the large value of the second cumulant. The decay times are roughly the same for both frequencies.

In Fig. 7 we show the behavior of the several SR quantifiers as functions of $D$. A comparison of Figs. 4 and 7 indicates that $Q_{u}, Q_{l}$, and $R_{\text {out }}$ have the same qualitative behavior for both frequencies. The nonmonotonic dependence on $D$ of $Q_{u}$ and $R_{\text {out }}$ is indicative of the existence of SR (for both frequencies) for the subthreshold input amplitude and in the ranges of $D$ values considered. The most relevant quantitative difference is that for $\Omega=0.1$ the SR gain remains less than unity.

\section{CONCLUSIONS}

With this work, we have analyzed the phenomenon of SR within the context of a noisy, bistable symmetric system

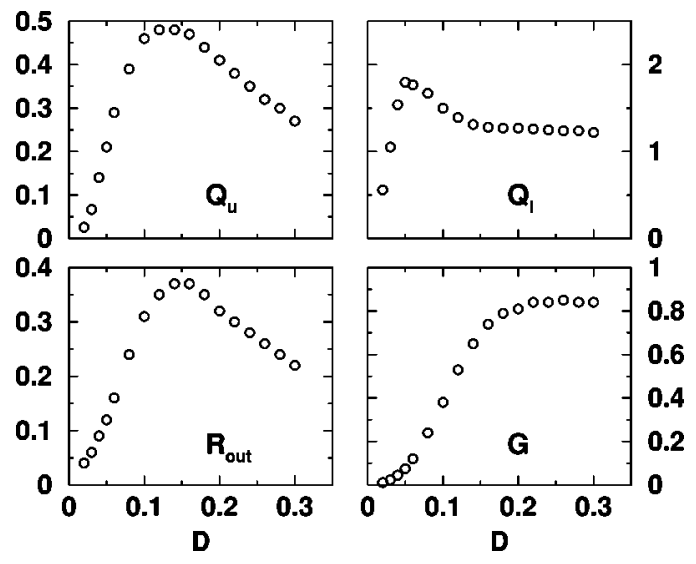

FIG. 7. Same as Fig. 4 but for $\Omega=0.1$.

driven by time-periodic, rectangular forcing possessing a duty cycle of unity. The numerical solution of the Langevin equation allows us to analyze the long-time behavior of the average, the second cumulant, and the coherent and incoherent parts of the correlation function. For subthreshold input signals we determined the SNR, together with its numerator and denominator evaluated separately, for a wide range of noise strengths $D$.

As a main result we find the simultaneous existence of a typical nonmonotonic behavior versus the noise strength $D$ of several quantifiers associated with SR; in particular, SR gains larger than unity are possible for a subthreshold rectangular forcing possessing a duty cycle of unity. This finding complements the recent results reported in Refs. [23,28], where pulselike signals with duty cycles smaller than or equal to 0.3 (see Fig. 7 in Ref. [23] and Fig. 8 in Ref. [28]) are studied and SR gains larger than unity are observed. Notice that in those references SR gains larger than unity are obtained only with input amplitudes larger than $0.8 A_{T}$. By contrast, in this work, we used a smaller input amplitude $\left(A \approx 0.68 A_{T}\right)$.

The simultaneous occurrence of SR and SR gains larger than unity is associated with the fact that, for some range of noise values, the decay time of the incoherent part of the correlation function is much shorter than $t_{a s y m}$ and also the probability distribution is basically monomodal during most of the cycle of the driving force.

\section{ACKNOWLEDGMENTS}

We acknowledge the support of the Dirección General de Enseñanza Superior of Spain (Grant No. BFM2002-03822), the Junta de Andalucía, the DAAD program "Acciones Integradas" (P.H., M.M.), and the Sonderforschungsbereich 486 (Project No. A10) of the Deutsche Forschungsgemeinschaft. 
[1] A. R. Bulsara and L. Gammaitoni, Phys. Today 49, 39 (1996).

[2] L. Gammaitoni, P. Hänggi, P. Jung, and F. Marchesoni, Rev. Mod. Phys. 70, 223 (1998).

[3] K. Wiesenfeld and F. Jaramillo, Chaos 8, 539 (1998).

[4] V. S. Anishchenko, A. B. Neiman, F. Moss, and L. Schimansky-Geier, Usp. Fiz. Nauk 169, 7 (1999).

[5] P. Hänggi, ChemPhysChem 3, 285 (2002).

[6] B. McNamara and K. Wiesenfeld, Phys. Rev. A 39, 4854 (1989).

[7] R. Kubo, J. Phys. Soc. Jpn. 12, 570 (1957).

[8] R. Kubo, Rep. Prog. Phys. 29, 255 (1966).

[9] P. Hänggi, Helv. Phys. Acta 51, 202 (1978).

[10] P. Hänggi and H. Thomas, Phys. Rep. 88, 207 (1982).

[11] P. Jung and P. Hänggi, Europhys. Lett. 8, 505 (1989).

[12] C. Presilla, F. Marchesoni, and L. Gammaitoni, Phys. Rev. A 40, 2105 (1989); L. Gammaitoni, E. Menichella-Saetta, S. Santucci, F. Marchesoni, and C. Presilla, ibid. 40, 2114 (1989).

[13] P. Jung and P. Hänggi, Phys. Rev. A 44, 8032 (1991).

[14] J. Casado-Pascual, J. Gómez-Ordóñez, M. Morillo, and P. Hänggi, Europhys. Lett. 58, 342 (2002).

[15] J. Casado-Pascual, J. Gómez-Ordóñez, M. Morillo, and P. Hänggi, Fluct. Noise Lett. 2, L127 (2002).

[16] M. I. Dykman, R. Mannella, P. V. E. McClintock, and N. G. Stocks, Phys. Rev. Lett. 68, 2985 (1992).

[17] P. Jung and P. Hänggi, Z. Phys. B: Condens. Matter 90, 255 (1993).

[18] F. Marchesoni, F. Apostolico, and S. Santucci, Phys. Lett. A 248, 332 (1998).
[19] M. I. Dykman, D. G. Luchinsky, R. Mannella, P. V. E. McClintock, N. D. Stein, and N. G. Stocks, Nuovo Cimento Soc. Ital. Phys. 17D, 660 (1995); M. DeWeese and W. Bialek, ibid. 17D, 733 (1995).

[20] J. Casado-Pascual, C. Denk, J. Gómez-Ordóñez, M. Morillo, and P. Hänggi, Phys. Rev. E 67, 036109 (2003).

[21] P. Hänggi, M. Inchiosa, D. Fogliatti, and A. R. Bulsara, Phys. Rev. E 62, 6155 (2000).

[22] K. Loerincz, Z. Gingl, and L. B. Kiss, Phys. Lett. A 224, 63 (1996); I. A. Khovanov and V. S. Anishenko, in Applied Nonlinear Dynamics and Stochastic Systems Near the Millenium, edited by J. B. Kadtke and A. Bulsara, AIP Conf. Proc. No. 411 (AIP, Woodbury, NY, 1997), pp. 267-272; Z. Gingl, R. Vajtai, and P. Makra, in Noise in Physical Systems and 1/f Fluctuations, edited by G. Bosman (World Scientific, Singapore, 2002), pp. 545-548.

[23] Z. Gingl, P. Makra, and R. Vajtai, Fluct. Noise Lett. 1, L181 (2001).

[24] P. Hänggi, P. Talkner, and M. Borkoveck, Rev. Mod. Phys. 62, 251 (1990).

[25] M. Morillo and J. Gómez-Ordóñez, Phys. Rev. E 51, 999 (1995).

[26] E. Helfand, Bell Syst. Tech. J. 58, 2289 (1979).

[27] H. S. Greenside and E. Helfand, Bell Syst. Tech. J. 60, 1927 (1981).

[28] P. Makra, Z. Gingl, and L. B. Kish, Fluct. Noise Lett. 2, L147 (2002). 